\title{
Serum D-serine levels are altered in early phases of Alzheimer's disease: towards a precocious biomarker
}

\author{
Luciano Piubelli $\mathbb{1 D}^{1}$, Loredano Pollegioni ${ }^{1}$, Valentina Rabattoni ${ }^{1}$, Marco Mauri ${ }^{1,2}$, Lucia Princiotta Cariddi ${ }^{3,4}$, \\ Maurizio Versino ${ }^{2,4}$ and Silvia Sacchi (1)
}

\begin{abstract}
D-Serine acts as a co-agonist of $\mathrm{N}$-methyl-D-aspartate receptors (NMDAR) which appear overactivated in AD, while Daspartate is a modulatory molecule acting on NMDAR as a second agonist. The aim of this work is to clarify whether the levels of these $D$-amino acids in serum are deregulated in AD, with the final goal to identify novel and precocious biomarkers in AD. Serum levels of $L$ - and D-enantiomers of serine and aspartate were determined by HPLC using a precolumn derivatization procedure and a selective enzymatic degradation. Experimental data obtained from agematched healthy subjects (HS) and AD patients were statistically evaluated by considering age, gender, and disease progression, and compared. Minor changes were apparent in the serum L- and D-aspartate levels in AD patients compared to HS. A positive correlation for the D-serine level and age was apparent in the AD cohort. Notably, the serum D-serine level and the D-/total serine ratio significantly increased with the progression of the disease. Gender seems to have a minor effect on the levels of all analytes tested. This work proposes that the serum D-serine level and $\mathrm{D}$-/total serine ratio values as novel and valuable biomarkers for the progression of AD: the latter parameter allows to discriminate CDR 2 and CDR 1 patients from healthy (CDR 0) individuals.
\end{abstract}

\section{Background}

Alzheimer's disease (AD), the most common cause of late-onset dementia, is a chronic and progressive neurodegenerative disease affecting $\approx 6 \%$ of adults over 65 years of age ${ }^{1}$. The preliminary diagnosis of $A D$ is made by a combination of clinical criteria, which includes neurological examinations, mental status tests, and brain imaging. On the basis of these clinical tests only, however, diagnosis of AD becomes a difficult task, especially in the early pre-symptomatic phase of the disease. Currently, analysis of the cerebrospinal fluid (CSF) with established biomarkers ${ }^{2,3}$ is carried out for research purposes. However,

Correspondence: Luciano Piubelli (luciano.piubelli@uninsubria.it)

'Department of Biotechnology and Life Sciences, University of Insubria, Varese, Italy

${ }^{2}$ Neurology Unit, Ospedale di Circolo and Fondazione Macchi, ASST Settelaghi, Varese, Italy

Full list of author information is available at the end of the article this fluid is obtained by an invasive and painful procedure: there is an extreme need for alternative, easily detectable biomarkers, which may also be sensitive and specific ${ }^{4}$.

$\mathrm{AD}$ pathophysiology is characterized by the accumulation of extracellular amyloid $\beta$ (A $\beta)$ plaques and intraneuronal inclusions of the truncated and phosphorylated forms of tau protein (neurofibrillary tangles). This appears to induce dystrophic neurites, loss of synapses, a prominent gliosis (involving changes in the morphology and function of microglia and astrocytes) and, only at later stages, overt loss of neurons and associated brain atrophy $^{5}$. Consistent with the role of the glutamatergic system in learning and memory formation, alterations in $N$-methyl-D-aspartate receptor (NMDAR)-mediated neurotransmission have been linked with the pathological processes underlying $A D^{6,7}$. Accordingly, $A \beta$ induces synaptic dysfunction by perturbing synaptic $\mathrm{Ca}^{2+}$ handling in response to overactivation of postsynaptic

\section{(c) The Author(s) 2021}

(c) (i) Open Access This article is licensed under a Creative Commons Attribution 4.0 International License, which permits use, sharing, adaptation, distribution and reproduction cc) in any medium or format, as long as you give appropriate credit to the original author(s) and the source, provide a link to the Creative Commons license, and indicate if changes were made. The images or other third party material in this article are included in the article's Creative Commons license, unless indicated otherwise in a credit line to the material. If material is not included in the article's Creative Commons license and your intended use is not permitted by statutory regulation or exceeds the permitted use, you will need to obtain permission directly from the copyright holder. To view a copy of this license, visit http://creativecommons.org/licenses/by/4.0/. 
NMDARs ${ }^{8}$, leading to oxidative stress, spine loss, and gradual neuronal cell death, which in turn correlates with the progressive decline in memory and cognition in $\mathrm{AD}^{7,9}$ but also by altering presynaptic functions ${ }^{10}$.

Among the major factors affecting NMDAR-mediated neurotransmission in $\mathrm{AD}$, availability of the agonist glutamate and the modulation of the receptor's functions are extremely important: ${ }^{11,12}$ the non-competitive NMDAR antagonist memantine has been approved for treatment of moderate to advanced $\mathrm{AD}^{13}$. Modulatory molecules playing a role in NMDAR function may also be related to AD. In particular, D-aspartate (D-Asp) acts as a second agonist ${ }^{14,15}$ and $\mathrm{D}$-serine (D-Ser) acts as the main endogenous co-agonist ${ }^{16}$.

In mammals, D-Asp is abundant in the embryonic brain, while during adulthood its levels are extremely low and strictly controlled by the catabolizing enzyme D-aspartate oxidase (DASPO $)^{17}$. The long-lasting exposure to nonphysiological, high concentration of D-Asp in DASPO knock-out mice $\left(\mathrm{Ddo}^{-/-}\right)$elicited a precocious decay of synaptic plasticity and cognitive functions ${ }^{18}$. Moreover, severe processes related to neuroinflammation were observed in this animal model, as indicated by the appearance of dystrophic microglia and reactive astrocytes $^{19}$, distinctive features in neurodegenerative disorders $^{20,21}$. Recently, the high D-Asp levels in $D d o^{-/-}$ mice were also shown to induce changes in tau phosphorylation $^{22}$. Notably, the D-Asp content in tissues and/ or biological fluids appeared to be altered in AD patients, despite differing levels having been reported: halved DAsp levels were detected in the white matter of AD brains compared to healthy subjects (HS) ${ }^{23}$ whereas higher levels were measured in the CSF of AD patients with respect to $\mathrm{HS}^{24,25}$. It is remarkable that all these studies were carried out on a limited number of subjects ( $\leq 10$ for both HS and $\mathrm{AD}$ patients).

On the other hand, brain D-Ser is synthesized starting from the corresponding L-enantiomer by the enzyme serine racemase (SR), and is degraded by both SR and Damino acid oxidase (DAAO, mainly located in astrocytes) ${ }^{26}$. Notably, a strong upregulation of SR was reported in reactive astrocytes in the hippocampus and entorhinal cortex of subjects with $\mathrm{AD}$, which increased with disease progression: large part of these astrocytes were also neurotoxic ${ }^{27}$. A $\beta$ aggregates induced release of $D-S e r$ and its brain content was increased in the animal models of the disease ${ }^{28-30}$, suggesting that the high D-Ser levels yield excitotoxicity, thus triggering neuronal death in AD. The DAAO inhibitor sodium benzoate improved cognitive and overall function in AD patients with earlyphase disease when used at high concentrations for 24 weeks ${ }^{31}$.

Concerning the detection of $\mathrm{D}$-Ser levels in AD patients, during the years contrasting results have been reported.
By using traditional high-performance liquid chromatography (HPLC) procedures, Nagata et al. ${ }^{32}$ reported no changes in D- and L-Ser levels in the frontal cortex compared to HS while a decrease in the D-Ser levels, coupled to a slight increase in L-Ser resulting in a significant decrease in D-/total-Ser ratio, was observed in human serum $^{33}$, albeit no details about the experimental procedure used are provided. Differently, an increase in D-Ser and SR levels was reported in post-mortem hippocampal and parietal cortex of AD patients compared to $\mathrm{HS}^{30}$. The same work reported higher D-Ser level in the CSF of probable $\mathrm{AD}$ than in non-cognitively impaired subject groups by using classical HPLC procedures. These results confirmed previous observations from D'Aniello's group ${ }^{25}$ performed using an HPLC method coupled to enzymatic degradation. Most recently, an analysis based on ultraHPLC-tandem mass spectrometry reported tiny changes in the D-Ser and D-/total-Ser levels ${ }^{34}$.

In our opinion, these confounding results are largely ascribable to the absence of standardized protocols and suitable controls. For this reason, this investigation is aimed at clarify whether D-Ser and D-Asp levels in serum are deregulated in AD using a well-established analytical procedure validated by the use of selective enzyme degradation and following the guidelines reported in ref. ${ }^{35}$. This with the final goal to propose D-Ser and/or DAsp as novel and precocious biomarkers in AD useful for detecting the progression of the disease.

\section{Materials and methods}

\section{Subject recruitment and sample collection}

Peripheral venous blood samples were collected from patients with $\mathrm{AD}$ and from age- and sex-matched $\mathrm{HS}$ recruited from outpatients attending the Alzheimer's Assessment Unit (CDCD) at the Ospedale di Circolo and Fondazione Macchi in Varese (ASST Settelaghi), Italy. AD was diagnosed according to the NIA-AD criteria ${ }^{3}$ and the disease stage of $\mathrm{AD}$ patients was assessed by using the Clinical Dementia Rating (CDR) Scale that allows to characterize five domains of cognitive and functional performance in AD dementia. Scores are defined as follows: 0: HS; 0.5 : questionable or very mild dementia; 1 : mild dementia; 2 : moderate dementia; 3 : severe dementia ${ }^{36}$. For this study, only $\mathrm{AD}$ patients presenting CDR 1 or 2 were enrolled. HS were caregivers of the recruited $\mathrm{AD}$ patients with MiniMental State Examination Score $\geq 27 / 30$ not suffering of present or past neurological and/or psychiatric pathologies influencing cognitive functions. The Ethics Committee of the Ospedale di Circolo and Fondazione Macchi of Varese approved the protocol and all participants signed a written informed consent before enrollment. Withdrawal of venous blood was performed after a fasting night, between 8:00 and 10:00 a.m., in BD Vacutainer ${ }^{\mathrm{TM}}$ SST $^{\mathrm{TM}}$ II Advances Tubes (Becton Dickinson, Franklin Lakes, NJ, USA) including clot 
activator and gel for serum separation. Tubes were coded and serum separation was performed by centrifugation. Sera were subsequently frozen and stored at $-80^{\circ} \mathrm{C}$ until HPLC analyses. The $\mathrm{AD}$ patients recruited for this study were not affected by serious behavioral disorders; therefore, they were not subjected to the administration of antipsychotic drugs.

\section{High-performance liquid chromatography analyses}

HPLC grade methanol, acetonitrile and tetrahydrofuran were from Honeywell International (Seelze, Germany). All other chemicals and reagents were from Merck Life Sciences (Darmstadt, Germany).

Serum samples were added of HPLC grade methanol $(90 \% \mathrm{v} / \mathrm{v}$ final concentration) and vigorously vortexed for $3 \mathrm{~min}$ at room temperature to precipitate serum proteins, that were then removed by centrifugation $(16,000 \times g$ for $15 \mathrm{~min}$ at $\left.4{ }^{\circ} \mathrm{C}\right)$. Supernatants were dried, suspended in $0.2 \mathrm{M}$ trichloroacetic acid, neutralized with $\mathrm{NaOH}$, and subjected to pre-column derivatization with o-phthalaldehyde and $\mathrm{N}$-acetyl-L-cysteine. Separation of the amino acid enantiomers was carried out by reversedphase HPLC on a Symmetry C8 column $(4.6 \times 250 \mathrm{~mm}$, bed volume $4 \mathrm{~mL}$ ) (Waters S.p.A., Sesto San Giovanni, MI, Italy) using a HPLC PU-2089 System (Jasco Europe, Cremella, LC, Italy) equipped with a fluorescence detector, as described in ref. ${ }^{37}$. Separation was carried out at $1 \mathrm{~mL} / \mathrm{min}$, under isocratic conditions in $0.1 \mathrm{M}$ sodium acetate in the presence of $1 \%$ ( $\mathrm{v} / \mathrm{v}$ final concentration) tetrahydrofuran at $\mathrm{pH} 6.2$ as the mobile phase. All investigated amino acids were detected in a single run lasting $30 \mathrm{~min}$. A washing step with $10 \mathrm{~mL}$ of $0.05 \mathrm{M}$ sodium acetate buffer, $47 \%$ acetonitrile and 3\% tetrahydrofuran (both $\mathrm{v} / \mathrm{v}$ final concentrations), $\mathrm{pH}$ 6.2, was performed after each run. Identification of D- and L-amino acids was based on retention times obtained with external standards (retention times of $4.3 \pm 0.2,5.1 \pm 0.2,21.2 \pm$ 0.6 , and $23.3 \pm 0.7 \mathrm{~min}$ for D-Asp, L-Asp, D-Ser, and L-Ser, respectively). Peaks identity was confirmed by the selective degradation of the D-enantiomers by RgDAAO M213R variant ${ }^{38}$ : the samples were added with $10 \mu \mathrm{g}$ of enzyme, incubated at $30^{\circ} \mathrm{C}$ for $4 \mathrm{~h}$, and then subjected to derivatization and HPLC analyses. Quantification of enantiomers was based on peak areas by means of calibration curves for each enantiomer.

Distribution of the values of variables was assessed by the D'Agostino and Pearson normality test. A nonnormal (or suspected non-normal) distribution for D-/LAsp and D-/L-Ser was observed, both as absolute levels and as ratios: therefore, a non-parametric approach was used for all statistical analyses. Statistical significance of two-samples comparisons of continuous variables were performed using the two-tailed Mann-Whitney test. Correlations among continuous variables were assessed by Spearman non-parametric correlation analyses. The values reported represent mean \pm standard deviation, unless otherwise stated. All analyses were performed using GraphPad Prism 7.0 (GraphPad Software, San Diego, CA, USA). A $P$ value $<0.05$ was considered as statistically significant.

\section{Results}

A total of $26 \mathrm{HS}$ from 64 to 86 years and $42 \mathrm{AD}$ patients from 64 to 87 years were enrolled for this study. The demographic and clinical characteristics of the subjects are reported in Table 1. The two groups are age-matched: the mean age was $76.7 \pm 5.9$ and $79.1 \pm 5.4$ years (mean \pm SD) for HS and AD patients, respectively: statistical analyses showed no significant differences $(P=0.0834$, Mann-Whitney test). Percentages of females were 53.8\% and $66.7 \%$ in HS and AD patients, respectively. Mean age values of male cohorts were $76.8 \pm 7.2$ and $78.5 \pm 5.4$ years (HS and AD patients, respectively), and those of female cohorts were $76.6 \pm 4.8$ and $79.4 \pm 5.4$ years (HS and AD patients, respectively): in these cases also no statistically significant differences have been found comparing mean age values of $\mathrm{HS}$ and $\mathrm{AD}$ patients $(P=0.4687$ and $P=0.0711$ for male and female cohorts, respectively, Mann-Whitney test).

The levels of D- and L-aspartate and D- and L-serine in serum were assayed by the pre-column derivatization procedure, confirming the identity and amount of the D-enantiomers (present at a significantly lower level) by a selective enzymatic degradation. Age-related variation of the investigated amino acids was analyzed by Spearman non-parametric correlation analyses (Supplementary Fig. 1): the D- and L-Asp levels and D-/total-Asp ratio did not significantly vary with age, neither in samples from HS nor from AD patients (D-Asp HS: $r=0.216, P=0.2891$; D-Asp AD: $r=0.02573, P=0.8715$; L-Asp HS: $r=0.03024, P=$ 0.8834; L-Asp AD: $r=-0.2589, P=0.8707$; D-/total-Asp ratio HS: $r=0.1133, P=0.5814$; D-/total-Asp ratio $\mathrm{AD}: r=$ $0.02715, P=0.8645)$. The same analyses showed no correlations with age for $\mathrm{D}-$ and $\mathrm{L}$-Ser levels and for $\mathrm{D}$-/total-Ser ratio in samples from HS (D-Ser: $r=0.1002, P=0.6264$; L-Ser: $r=-0.2784, P=0.1684$; D-/total-Ser ratio: $r=$ $0.3844, P=0.525$ ); while a positive correlation has been found both for D-Ser $(r=0.5242, P=0.0004)$ and for D-/ total-Ser ratio $(r=0.3663, P=0.0170)$, but not for $\mathrm{L}-\mathrm{Ser}$ $(r=0.2164, P=0.1687)$, in samples from AD patients.

In order to exclude any effect due to the administration of memantine on the levels of the analyzed molecules, the values obtained for patients assuming and not assuming the drug were compared. In all cases, no significant difference has been observed (D-Ser: $P=0.6753$; L-Ser: $P=$ 0.4872; D-/total-Ser ratio: $P=0.7977$; D-Asp: $P=0.6570$; L-Asp: $\quad P=0.0918 ; \quad$ D-/total-Asp ratio: $\quad P=0.3126$; Mann-Whitney test). Thus, all patients were considered as a single group. 
Table 1 Demographic and clinical data of the subjects enrolled for the study.

\begin{tabular}{|c|c|c|c|}
\hline Demographic data & Healthy subjects & $A D$ patients & $P$ value \\
\hline Number & 26 & 42 & - \\
\hline Male (\% of total) & $12(46.2)$ & $14(33.3)$ & $0.2904^{*}$ \\
\hline Female (\% of total) & $14(53.8)$ & $28(66.7)$ & \\
\hline Age, range (years) & $64-86$ & $64-87$ & - \\
\hline Male, range (years) & $64-86$ & $64-87$ & - \\
\hline Female, range (years) & $66-84$ & $68-86$ & - \\
\hline Age, mean $\pm S D(S E M)$ & $76.7 \pm 5.9(1.16)$ & $79.1 \pm 5.4(0.83)$ & 0.0834 \\
\hline Male, age, mean \pm SD (SEM) & $76.8 \pm 7.2(2.08)$ & $78.5 \pm 5.4(1.44)$ & 0.4687 \\
\hline Female, age, mean \pm SD (SEM) & $76.6 \pm 4.8(1.28)$ & $79.4 \pm 5.4(1.03)$ & 0.0711 \\
\hline $\mathrm{CDR}$, mean $\pm \mathrm{SD}(\mathrm{SEM})$ & NA & $1.41 \pm 0.50(0.077)$ & - \\
\hline Male, CDR, mean \pm SD (SEM) & NA & $1.43 \pm 0.51(0.14)$ & - \\
\hline Female, CDR, mean \pm SD (SEM) & NA & $1.39 \pm 0.50(0.094)$ & - \\
\hline \multicolumn{4}{|l|}{$\mathrm{CDR}$, number (in parentheses) and age, mean $\pm \mathrm{SD}$ (SEM) } \\
\hline CDR 1 (25) & NA & $77.9 \pm 5.7(1.15)$ & \multirow[t]{2}{*}{$0.1012^{* *}$} \\
\hline CDR 2 (17) & NA & $80.9 \pm 4.4(1.06)$ & \\
\hline No. of subjects using anti-dementia drugs (\% of total) $)^{a}$ & NA & $38(90.5)$ & \\
\hline Memantine, number (\% of total) & NA & $19(42.9)$ & - \\
\hline Memantine, mg/die, mean \pm SD (SEM) & NA & $18.4 \pm 3.8(0.86)$ & - \\
\hline Donepezil, number (\% of total) & NA & $14(33.3)$ & - \\
\hline Donepezil, mg/die, mean \pm SD (SEM) & NA & $7.5 \pm 2.6(0.69)$ & - \\
\hline Rivastigmine, number (\% of total) & NA & $7(16.7)$ & - \\
\hline Rivastigmine, mg/die, mean \pm SD (SEM) & NA & $6.6 \pm 2.1(0.79)$ & - \\
\hline
\end{tabular}

SD standard deviation, SEM standard error of the mean; $P$ values refer to comparison between HS and AD patients (Mann-Whitney test). NA not applicable. *The comparison was performed using the $X^{2}$ test.

${ }^{* *} P$ value refers to the comparison between CDR 1 and CDR 2 AD patients (Mann-Whitney test). $P$ values obtained from comparison between HS and AD patients disaggregated by CDR are reported in the text.

${ }^{\mathrm{a}}$ Two AD patients were assuming both memantine and donepezil.

Levels of D-Asp were only slightly decreased in AD patients compared to HS $(-2.47 \%)$, whereas an increase $(+25.3 \%)$ was observed for L-Asp: consequently, a decrease $(-28.7 \%)$ was observed for the D-/total-Asp ratio (Fig. 1 and Supplementary Table 1). These variations resulted not statistically significant. Analyzing the data disaggregated by gender, significant variations between HS and AD patients were found only for the male cohort, in the case of L-Asp and of D-/total-Asp ratio $(P=0.0178$ and 0.0464 , respectively). Minor and no statistically significant differences were found for female cohort. Comparing data of male and female subjects belonging to the same group of subjects (Fig. 2 and Supplementary Table 1), a statistically significant difference was apparent for HS and L-Asp only $(P=$ $0.0178)$, and the relative $\mathrm{D}-/$ total-Asp ratio $(P=0.0464)$. The levels of Asp enantiomers were not affected by gender in $\mathrm{AD}$ patients.
The D-Ser level showed a statistically significant increase in $\mathrm{AD}$ patients with respect to $\mathrm{HS}(+21.8 \%, P=$ 0.0060), whereas the L-Ser level was unchanged (Fig. 1B). Similar to D-Ser, a statistically significant increase was observed for the D-/total-Ser ratio $(+20.2 \%, P=0.0025)$ (Fig. 1 and Supplementary Table 1). A similar pattern was observed comparing cohorts disaggregated by gender: in female cohorts the D-Ser level increased by $28.7 \%$, the D- 1 total-Ser ratio increased by $31.4 \%$, and the $\mathrm{L}$-Ser level was unchanged. In male cohorts, a lower increase of D-Ser and of D-/total-Ser ratio was observed $(+12.3 \%$ and $+11.2 \%$, respectively) whereas the L-Ser level was unchanged. Comparison between male and female subjects of the same group (Fig. 2 and Supplementary Table 1) revealed a statistical significance only for D-/total-Ser ratio $(P=0.0032)$ of the HS subjects. Similar to D-Asp, gender did not significantly affect the level of Ser enantiomers in $\mathrm{AD}$ patients. 

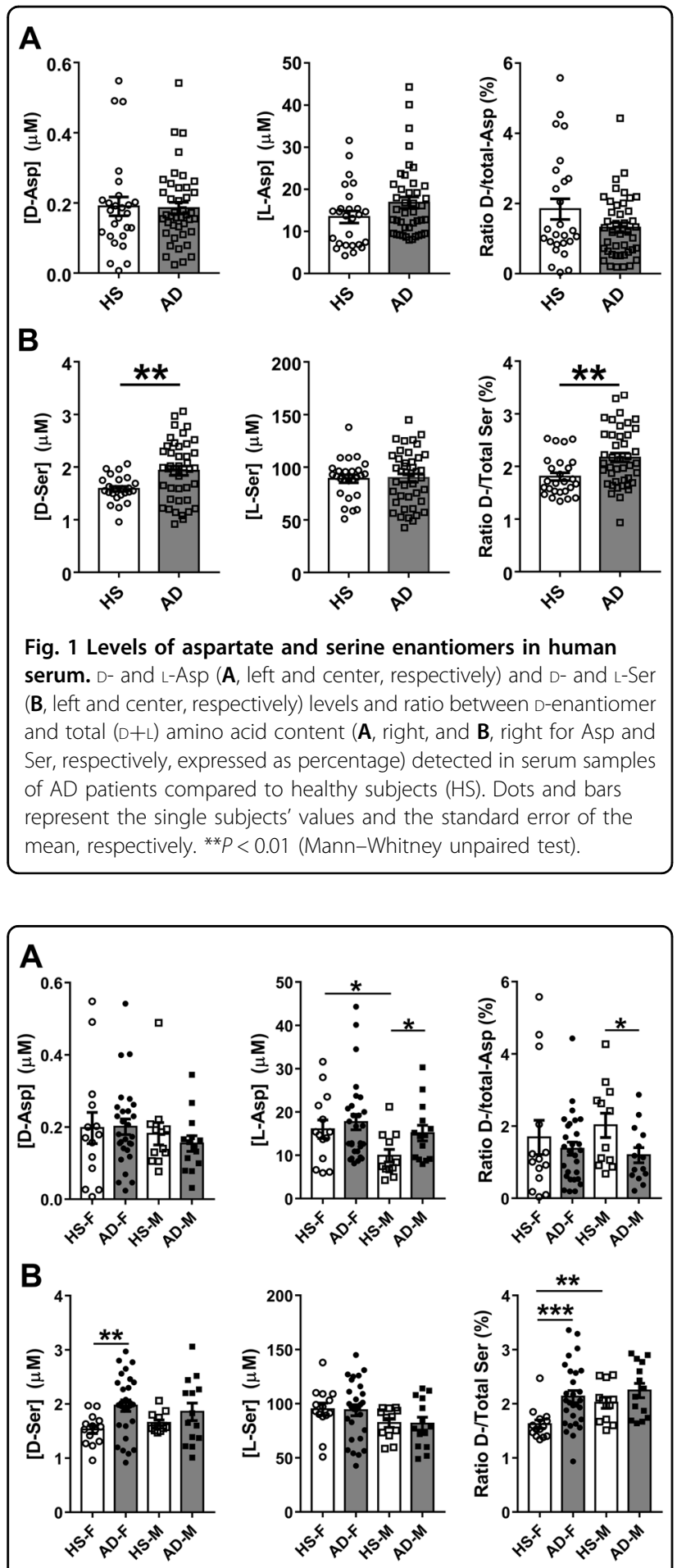

Fig. 2 Gender related variations of aspartate and serine enantiomer levels in human serum. D- and L-Asp (A, left and center, respectively) and D- and L-Ser (B, left and center, respectively) levels and ratio between $\mathrm{D}$-enantiomer and total $(\mathrm{D}+\mathrm{L})$ amino acid content $(\mathbf{A}$ right and $\mathbf{B}$ right for Asp and Ser, respectively, expressed as percentage) detected in serum samples of AD patients compared to healthy subjects (HS). Dots and bars represent the single subjects' values and the standard error of the mean, respectively. ${ }^{*} P<0.05$; ${ }^{* *} P<0.01 ;{ }^{* *} P<$ 0.001 (Mann-Whitney unpaired test). F female, M male.
Table 2 Statistical analysis of the levels of aspartate and serine enantiomers, D-/total-Asp and D-/total-Ser ratios observed in serum of HS and AD patients.

\begin{tabular}{|c|c|c|c|c|c|c|}
\hline \multirow[t]{2}{*}{ Amino acid } & \multicolumn{2}{|c|}{ CDR 1 vs. CDR 0} & \multicolumn{2}{|c|}{ CDR 2 vs. CDR 1} & \multicolumn{2}{|c|}{ CDR 2 vs. CDR 0} \\
\hline & $\Delta \%$ & $P$ value & $\Delta \%$ & $P$ value & $\Delta \%$ & $P$ value \\
\hline D-Asp & 1.58 & 0.6040 & -9.84 & 0.4733 & -8.42 & 0.9072 \\
\hline$-A s p$ & 23.1 & 0.2431 & 4.85 & 0.2287 & 29.1 & 0.0787 \\
\hline Ratio D-/total-Asp & -19.6 & 0.7017 & -27.7 & 0.2389 & -41.8 & 0.0977 \\
\hline D-Ser & 15.9 & 0.0873 & 12.5 & 0.2388 & 30.2 & 0.0015 \\
\hline L-Ser & 0.79 & 0.7156 & 0.33 & 0.8443 & 1.12 & 0.9070 \\
\hline Ratio D-/total-Ser & 19.4 & 0.0336 & 10.1 & 0.2400 & 26.5 & 0.0016 \\
\hline
\end{tabular}

$C D R \quad 0$ healthy subjects, $C D R 1$ mild dementia, CDR 2 moderate dementia. $\triangle \%$ variation between compared groups expressed as percentage $(\triangle \%=100 \times$ $\left([C D R]_{n}-[C D R]_{n-1}\right) /[C D R]_{n-1}$, where " $n$ " is the CDR score, for CDR 1 vs. CDR 0 and CDR 2 vs. CDR 1 , and $\Delta \%=100 \times\left([C D R]_{2}-[C D R]_{0}\right) /[C D R]_{0}$ for CDR 2 vs. CDR 0$)$. $P$ values obtained with non-parametric Mann-Whitney test are indicated for each comparison. Statistically significant $P$ values $(P<0.05)$ are in bold.

D-Serine levels increase with the progression of the disease in the early stages

The levels of aspartate and serine enantiomers have been compared between homogeneous groups regarding the stage of illness, assessed by the CDR score ${ }^{39}$. AD patients enrolled in this study were at initial stages of $A D$, corresponding to mild (CDR 1) or moderate (CDR 2) dementia.

Considering the aspartate levels, comparison of mean levels of analyzed parameters observed for HS, CDR 1, and CDR 2 AD patients did not show any statistical significance (Table 2 and Fig. 3A). However, a modest increase parallel to the gravity of the pathology has been observed for L-Asp together with a parallel decrease in D-/ total-Asp ratio (Table 2 and Fig. 3A).

Analyses of the results obtained for serine clearly show an increase in the D-Ser levels with the severity of the pathology: statistically significant differences have been found between HS and CDR 2 AD patients' mean values $(+30.2 \%, P=0.0015)$ (Table 2 and Fig. 3B, left). Since the L-Ser levels remain unchanged (Table 2 and Fig. 3B, center), an increase in the $\mathrm{D}$-/total-Ser ratio was observed: statistically significant variations of this parameter have been found both between HS (CDR 0) and CDR 1 AD patients $(+19.4 \%, P=0.0336)$ and between HS and CDR 2 AD patients $(+26.5 \%, P=0.0016)$ (Table 2 and Fig. $3 \mathrm{~B}$, right). Analysis of age distribution of $\mathrm{HS}, \mathrm{CDR} 1$, and CDR 2 AD patients, carried out using Mann-Whitney test, indicated that a statistically significant difference was observed between HS and CDR 2 AD patients only $(P=$ 0.0105), whereas comparison between HS and CDR 1 AD patients and CDR 1 and CDR 2 AD patients was not statistically significant $(P=0.5077$ and $P=0.1012$, 


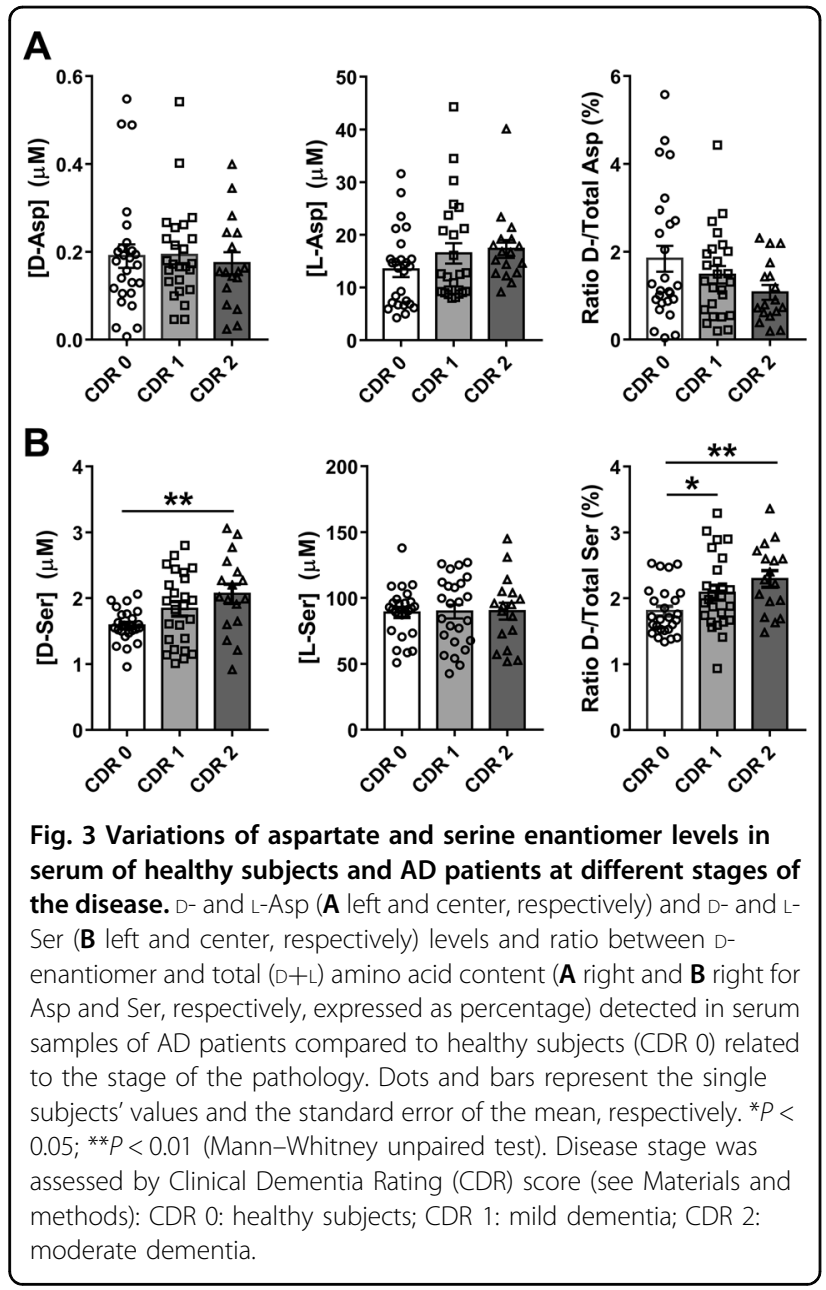

respectively, see Table 1 ). These results exclude that the observed increase in the D-Ser serum level was due to differences in age of the cohorts analyzed.

\section{Discussion}

$\mathrm{AD}$ is the most common type of dementia in aged people: the pathological changes associated with $\mathrm{AD}$ (amyloid deposition and the resulting neuronal death) start decades before the first clinical symptoms appear. Thus, it is crucial to identify and detect parameters indicative of neuropathological changes, eventually those occurring at the synaptic level, in the very precocious stages of the disease. Diagnosis is now based on a neuropsychological evaluation and the assessment of $\mathrm{AD}$ biomarkers, such as $A \beta$ oligomers and phosphorylatedtau levels in the CSF. However, CSF examinations are far from standard tests in general practice, due to the invasiveness of the procedure (a lumbar puncture), which also limits this practice for screening and clinical trials. Therefore, there is an urgent need for alternative, accessible peripheral biomarkers, such as serum biomarkers. Accordingly, we monitored the levels of D-Asp and D-Ser, two signaling molecules involved in NMDAR-mediated neurotransmission, in serum from $\mathrm{AD}$ patients at early stages of illness (CDR 1 or 2) and age-matched controls (CDR 0). We employed a standardized, well-validated analytical procedure based on pre-column derivatization, HPLC separation, and quantification of the D-enantiomers by enzymatic degradation.

D-Asp and L-Asp serum content did not show any statistically significant variation between $\mathrm{AD}$ patients and $\mathrm{HS}$, despite a reasonable increase in L-Asp levels $(\approx 25 \%)$ was observed in $\mathrm{AD}$ patients. By analyzing data disaggregated by gender, statistically significant differences were observed for L-Asp only: in the HS male cohort the L-Asp levels were significantly lower compared to the corresponding $\mathrm{AD}$ cohort and to female ones (independently from the diagnosis). As a consequence, in the $\mathrm{AD}$ male cohort the $\mathrm{D}-1$ total-Asp ratio appeared significantly decreased compared to controls. On the other hand, statistically significantly elevated D-Ser serum levels $(\approx 20 \%)$ were detected in $\mathrm{AD}$ patients with respect of HS. Data disaggregated by gender indicate a higher increase in female cohorts $(\approx 30 \%)$ compared to male $(\approx 10 \%)$ cohorts, despite in this latter case the difference between $\mathrm{AD}$ patients and HS was not statistically significant. No significant change depending on diagnosis and/or gender is observed in L-Ser serum levels, while the D-/total-Ser ratio is higher in male compared to female HS cohorts. Overall, our results point to a slight gender effect on the serum levels of both D- and L-Asp and D- and L-Ser, thus confirming previous results obtained in ventricular $\mathrm{CSF}^{25}$ and in post mortem tissues and $\mathrm{CSF}^{30}$. On the other hand, a significant positive correlation between serum D-serine levels and age has been observed in AD patients and not in HS, despite a negative association previously reported ${ }^{39}$.

Separating subjects into experimental groups stratified by the CDR score showed an increasing trend for L-Asp content from cognitively HS (CDR 0) to patients with a CDR score of 2, which resulted into a specular trend in the D-/total-Asp ratio values. Lack of statistical significance for D-Asp might be ascribed to its very low concentration $(0.15-0.2 \mu \mathrm{M})$, close to the limit of detection. Conversely, serum D-Ser levels paralleled the progression of the pathology: the lowest levels were detected in HS and increasing levels were observed in AD patients, being significantly higher in individuals with a CDR of 2 . This positive correlation is even more evident for D-/totalSer ratio. In this latter case, a statistically significant increase is also evident in patients with a CDR score of 1 , suggesting this ratio as a more sensitive parameter of the pathology and its progression than absolute D-Ser levels.

This latter result is in line with recent observations reporting increased D-serine levels in some areas of $\mathrm{AD}$ brains involved in the disease progression ${ }^{30}$, likely due to upregulation of SR expression in reactive neurotoxic astrocytes $^{27}$ and the consequent increased D-Ser release. 
Worthy of note, converging lines of evidence indicate reduced levels of NMDAR in the same brain regions in $\mathrm{AD}$ patients ${ }^{40,41}$. It has been suggested that elevated levels of the co-agonist D-Ser might underlie a mechanism by which $A \beta$ oligomers trigger synapse dysfunction and the resulting memory impairment ${ }^{30}$ : $A \beta$-induced $D$-Ser increase might represent an initial adaptive response to maintain proper neurotransmission in the early stages of the disease ${ }^{42}$, but, since NMDARs appear overactivated in $\mathrm{AD}$, they can also contribute to the excitotoxic scenario in later stages, worsening the neuropathological outcomes. D-Ser inhibits apoptosis at early phases and stimulates necrosis at later phases ${ }^{43}$. The development of amyloid plaques typically precedes clinically significant cognitive symptoms by at least $10-15$ years: a time course comparison between plaques formation and D-Ser levels could allow to elucidate the events bringing to the dementia. Future studies should also evaluate the correlation between $\mathrm{D}-$ Ser levels and peripheral biomarkers of $\mathrm{AD}$, i.e. blood levels of $A \beta 42$, phosphorylated tau, etc.

As a next step in the field, analyses on subjects showing amnestic mild cognitive impairment $(\mathrm{MCI}, \mathrm{CDR}=0.5)$ and with a significantly increased probability to develop AD will allow to validate the use of serum serine levels as a valuable biomarker of the disease onset. To be enrolled in this study, amnestic MCI patients need to undergo imaging analysis (e.g., PET) to verify the presence of a neurodegenerative process.

\section{Conclusions}

Considerations related to the effect of $A \beta$ on the D-Ser synthesis/level, and thus on NMDAR functioning, in AD pathophysiology and our results concerning serine levels (determined by a procedure based on enzymatic selective degradation of the D-enantiomer) strengthen the idea of serum D-Ser levels and/or D-/total-Ser ratio as a valuable (and simple to assay) biological marker for $\mathrm{AD}$ to evaluate the disease progression as well as the precocious stages of the illness. Accordingly, we suggest the combined use of blood-based biomarkers currently under development ${ }^{44}$ and serine enantiomers concentration ratio as a novel and relevant strategy to increase sensitivity and specificity in the diagnosis of $\mathrm{AD}$.

\section{Acknowledgements}

This work was supported by grant from Fondo di Ateneo to L. Piubelli, S.S., and L. Pollegioni. V.R. is a Ph.D. student of the Biotechnology and Life Sciences course at the University of Insubria. L.P.C. is a Ph.D. student of the Clinical and Experimental Medicine and Medical Humanities course at the University of Insubria.
}

\footnotetext{
Author details

${ }^{1}$ Department of Biotechnology and Life Sciences, University of Insubria, Varese, Italy. ${ }^{2}$ Neurology Unit, Ospedale di Circolo and Fondazione Macchi, ASST Settelaghi, Varese, Italy. ${ }^{3}$ Center of Research in Medical Pharmacology, University of Insubria, Varese, Italy. ${ }^{4}$ Department of Medicine and Surgery, University of Insubria, Varese, Italy
}

Conflict of interest

The authors declare that they have no conflict of interest.

\section{Publisher's note}

Springer Nature remains neutral with regard to jurisdictional claims in published maps and institutional affiliations.

Supplementary information The online version contains supplementary material available at https://doi.org/10.1038/s41398-021-01202-3.

Received: 12 May 2020 Revised: 28 September 2020 Accepted: 20 October 2020

Published online: 26 January 2021

\section{References}

1. Qiu, C., Kivipelto, M. \& von Strauss, E. Epidemiology of Alzheimer's disease: occurrence, determinants, and strategies toward intervention. Dialogues Clin. Neurosci. 11, 111-128 (2009).

2. Jack, C. R. et al. Introduction to the recommendations from the National Institute on Aging-Alzheimer's Association workgroups on diagnostic guidelines for Alzheimer's disease. Alzheimers Dement. https://doi.org/10.1016/j. jalz.2011.03.004 (2011).

3. McKhann, G. M. et al. The diagnosis of dementia due to Alzheimer's disease: recommendations from the National Institute on Aging-Alzheimer's Association workgroups on diagnostic guidelines for Alzheimer's disease. Alzheimers Dement. https://doi.org/10.1016/j.jalz.2011.03.005 (2011).

4. Humpel, C. Identifying and validating biomarkers for Alzheimer's disease. Trends Biotechnol. https://doi.org/10.1016/j.tibtech.2010.09.007 (2011).

5. Danysz. W. \& Parsons, C. G. Alzheimer's disease, $\beta$-amyloid, glutamate, NMDA receptors and memantine-searching for the connections. Br. J. Pharmacol. https://doi.org/10.1111/j.1476-5381.2012.02057.x (2012).

6. Francis, P. T. Glutamatergic systems in Alzheimer's disease. Int. J. Geriatr. Psychiatry https://doi.org/10.1002/gps.934 (2003).

7. Wenk, G. L. Neuropathologic changes in Alzheimer's disease: potential targets for treatment. J. Clin. Psychiatry 3, 3-7 (2006).

8. Wang, R., Reddy, P. H. Role of glutamate and NMDA teceptors in Alzheimer's fisease. J. Alzheimers Dis. https://doi.org/10.3233/JAD-160763 (2017).

9. Danysz, W. \& Parsons, C. G. The NMDA receptor antagonist memantine as a symptomatological and neuroprotective treatment for Alzheimer's disease: preclinical evidence. Int. J. Geriatr. Psychiatry https://doi.org/10.1002/gps.938 (2003).

10. Barthet, G. \& Mulle, C. Presynaptic failure in Alzheimer's disease. Prog. Neurobiol. https://doi.org/10.1016/j.pneurobio.2020.101801 (2020).

11. Scott, H. A., Gebhardt, F. M., Mitrovic, A. D., Vandenberg, R. J. \& Dodd, P. R. Glutamate transporter variants reduce glutamate uptake in Alzheimer's disease. Neurobiol. Aging https:/doi.org/10.1016/.jneurobiolaging.2010.03.008 (2011).

12. Talantova, M. et al. AB induces astrocytic glutamate release, extrasynaptic NMDA receptor activation, and synaptic loss. Proc. Natl. Acad. Sci. USA https:// doi.org/10.1073/pnas.1306832110 (2013).

13. Rudy, C. C., Hunsberger, H. C., Weitzner, D. S. \& Reed, M. N. The role of the tripartite glutamatergic synapse in the pathophysiology of Alzheimer's disease. Aging Dis. https://doi.org/10.14336/AD.2014.0423 (2015)

14. Errico, F., Napolitano, F., Nisticò, R. \& Usiello, A. New insights on the role of free D-Asp in the mammalian brain. Amino Acids https://doi.org/10.1007/s00726012-1356-1 (2012).

15. Krashia, P. et al. Persistent elevation of D-aspartate enhances NMDA receptor-mediated responses in mouse substantia nigra pars compacta dopamine neurons. Neuropharmacology https://doi.org/10.1016/j. neuropharm.2015.12.013 (2016).

16. Mothet, J.P. et al. D-Serine is an endogenous ligand for the glycine site of the N-methyl-D-aspartate receptor. Proc. Natl. Acad. Sci. USA https://doi.org/ 10.1073/pnas.97.9.4926 (2000).

17. Schell, M. J., Cooper, O. B. \& Snyder, S. H. D-aspartate localizations imply neuronal and neuroendocrine roles. Proc. Natl. Acad. Sci. USA https://doi.org/ 10.1073/pnas.94.5.2013 (1997).

18. Errico, F. et al. Persistent increase of D-aspartate in D-aspartate oxidase mutant mice induces a precocious hippocampal age-dependent synaptic plasticity 
and spatial memory decay. Neurobiol. Aging https://doi.org/10.1016/j. neurobiolaging.2009.12.007 (2011).

19. Punzo, D. et al. Age-related changes in D-aspartate oxidase promoter methylation control extracellular D-aspartate levels and prevent precocious cell death during brain aging. J. Neurosci. https://doi.org/10.1523/ JNEUROSCI.3881-15.2016 (2016)

20. Liddelow, S. A. et al. Neurotoxic reactive astrocytes are induced by activated microglia. Nature https://doi.org/10.1038/nature21029 (2017).

21. Yun, S. P. et al. Block of A1 astrocyte conversion by microglia is neuroprotective in models of Parkinson's disease. Nat. Med. https://doi.org/10.1038/ s41591-018-0051-5 (2018).

22. Nuzzo, T. et al. Free D-aspartate triggers NMDA receptor-dependent cell death in primary cortical neurons and perturbs JNK activation, Tau phosphorylation, and protein SUMOylation in the cerebral cortex of mice lacking D-aspartate oxidase activity. Exp. Neurol. https://doi.org/10.1016/j.expneurol.2019.02.014 (2019).

23. Fisher, G. H. et al. Free D-aspartate and D-alanine in normal and Alzheimer brain. Brain Res. Bull. https://doi.org/10.1016/0361-9230(91)90266-m (1991).

24. Fisher, G. H. et al. Free D-amino acids in human cerebrospinal fluid of Alzheimer disease, multiple sclerosis, and healthy control subjects. Mol. Chem. Neuropathol. https://doi.org/10.1007/bf02815405 (1994).

25. Fisher, G. et al. Free D- and L-amino acids in ventricular cerebrospinal fluid from Alzheimer and normal subjects. Amino Acids https://doi.org/10.1007/ bf01318865 (1998).

26. Pollegioni, L. \& Sacchi, S. Metabolism of the neuromodulator D-serine. Cell. Mol. Life Sci. https:/doi.org/10.1007/s00018-010-0307-9 (2010).

27. Balu, D. T. et al. Neurotoxic astrocytes express the D-serine synthesizing enzyme, serine racemase, in Alzheimer's disease. Neurobiol. Dis. https://doi.org/ 10.1016/j.nbd.2019.104511 (2019)

28. Wu, S.Z. et al. Induction of serine racemase expression and D-serine release from microglia by amyloid beta-peptide. J. Neuroinflammation https://doi.org/ 10.1186/1742-2094-1-2 (2004).

29. Brito-Moreira, J. et al. A $\beta$ oligomers induce glutamate release from hippocampal neurons. Curr. Alzheimer Res. https://doi.org/10.2174/ 156720511796391917 (2011).

30. Madeira, C. et al. D-serine levels in Alzheimer's disease: implications for novel biomarker development. Transl. Psychiatry https://doi.org/10.1038/tp.2015.52 (2015).

31. Lin, C. H. et al. Benzoate, a D-amino acid oxidase inhibitor, for the treatment of early-phase Alzheimer disease: a randomized, double-blind, placebo-controlled trial. Biol. Psychiatry https://doi.org/ 10.1016/j.biopsych.2013.08.010 (2014).

32. Nagata, Y., Borghi, M., Fisher, G. H. \& D'Aniello, A. Free D-serine concentration in normal and Alzheimer human brain. Brain Res. Bull. https://doi.org/10.1016/ 0361-9230(95)00087-u (1995).

33. Hashimoto, K. et al. Possible role of D-serine in the pathophysiology of $\mathrm{Alz}$ heimer's disease. Prog. Neuropsychopharmacol. Biol. Psychiatry https://doi.org/ 10.1016/j.pnpbp.2003.11.009 (2004).

34. Biemans, E. A. et al. CSF D-serine concentrations are similar in Alzheimer's disease, other dementias, and elderly controls. Neurobiol. Aging. https://doi. org/10.1016/j.neurobiolaging.2016.03.017 (2016).

35. Mothet, J.P., Billard, J.-M., Pollegioni, L., Coyle, J. T. \& Sweedler, J. V. Investigating brain D-serine: advocacy for good practices. Acta Physiol. (Oxf.) https:/ doi.org/10.1111/apha.13257 (2019).

36. Hughes, C. P., Berg, L., Danziger, W. L., Coben, L. A. \& Martin, L. R. A new clinical scale for the staging of dementia. Br. J. Psychiatry https://doi.org/10.1192/ bjp.140.6.566 (1982).

37. Nuzzo, T. et al. Decreased free D-aspartate levels are linked to enhanced Daspartate oxidase activity in the dorsolateral prefrontal cortex of schizophrenia patients. npj Schizophr. https://doi.org/10.1038/s41537-017-0015-7 (2017).

38. Sacchi, S. et al. Engineering the substrate specificity of D-amino-acid oxidase. J. Biol. Chem. https://doi.org/10.1074/jbc.M203946200 (2002).

39. Calcia, M. A. et al. Plasma levels of D-serine in Brazilian individuals with schizophrenia. Schizophr. Res. https://doi.org/10.1016/.jschres.2012.09.014 (2012).

40. Hynd, M. R., Scott, H. L. \& Dodd, P. R. Differential expression of N-methyl-Daspartate receptor NR2 isoforms in Alzheimer's disease. J. Neurochem. https:// doi.org/10.1111/j.1471-4159.2004.02548.x (2004).

41. Mishizen-Eberz, A. J. et al. Biochemical and molecular studies of NMDA receptor subunits NR1/2A/2B in hippocampal subregions throughout progression of Alzheimer's disease pathology. Neurobiol. Dis. https:/doi.org/ 10.1016/.j.nbd.2003.09.016 (2004).

42. Guercio, G. D. \& Panizzutti, R. Potential and challenges for the clinical use of Dserine as a cognitive enhancer. Front. Psychiatry https://doi.org/10.3389/ fpsyt.2018.00014 (2018).

43. Esposito, S. et al. Contribution of serine racemase/D-serine pathway to neuronal apoptosis. Aging Cell https://doi.org/10.1111/j.1474-9726.2012.00822.x (2012).

44. Hampel, H. et al. Blood-based biomarkers for Alzheimer disease: mapping the road to the clinic. Nat. Rev. Neurol. https://doi.org/10.1038/s41582-018-0079-7 (2018). 